\title{
Análisis bibliométrico de las publicaciones peruanas relacionadas a resistencia antimicrobiana en SCOPUS (1992-2017)
}

\author{
Elizabeth Espinoza-Portilla ${ }^{1, a}$, Flor Lioo-Jordán ${ }^{2, b}$, Gladis J. Villanueva-Cadenas ${ }^{3, c}$
}

\section{RESUMEN}

La resistencia antimicrobiana es un fenómeno generalizado que puede afectar a cualquier persona sin distinguir edad o país de procedencia. Hasta la fecha no existen estudios bibliométricos peruanos sobre resistencia antimicrobiana. Este estudio se llevó a cabo para analizar y presentar indicadores bibliométricos relacionados con las publicaciones peruanas sobre resistencia antimicrobiana. La recolección de datos se llevó a cabo utilizando la base de datos Scopus. Se recuperaron un total de 34 artículos. Las publicaciones aumentaron considerablemente a partir del año 2015, y llegaron a un récord de 11 el año 2017. De estas, la mayoría fueron artículos originales, redactados con más frecuencia, en inglés. Las publicaciones se originaron en diferentes regiones del mundo, incluidas América del Norte, Europa y América Latina. La mayor parte de los artículos recuperados se publicaron en revistas prestigiosas de biología, medicina, microbiología y en revistas relacionadas con el control de infecciones.

Palabras clave: Farmacorresistencia microbiana; Resistencia a medicamentos; Productos con acción antimicrobiana; Bibliometría; Perú (Fuente: DeCS BIREME).

\section{Bibliometric analysis of Peruvian publications on antimicrobial resistance in Scopus database (1992-2017)}

\section{ABSTRACT}

Antimicrobial resistance is a generalized phenomenon that can affect people from any age or country. To date, there are no Peruvian bibliometric studies on antimicrobial resistance. This study was carried out to analyze and present bibliometric indicators related to Peruvian publications on antimicrobial resistance. Data collection was performed using the Scopus database. A total of 34 articles were retrieved. Peruvian publications on antimicrobial resistance increased considerably since 2015, reaching a record of 11 in 2017 . Out of these, most of the documents were original articles and the most frequent language was English. Publications originated in different parts of the world, including North America, Europe and Latin America. Most of the retrieved articles were published in prestigious journals of biology, medicine and microbiology, and journals related to infection control.

Keywords: Drug resistance, microbial; Drug resistance; Products with antimicrobial action; Bibliometrics; Peru (Source: MeSH NLM).

1. Universidad de San Martin de Porres, Instituto de Gobierno y de Gestión Pública. Lima, Perú.

2. Universidad Nacional José Faustino Sánchez Carrión. Huacho, Perú.

3. Universidad Nacional José Faustino Sánchez Carrión, Facultad de Medicina Humana, Escuela de Enfermería. Huacho, Perú.

a. Doctora en Gobierno y Políticas Públicas, Magíster en Estudios de Políticas Públicas, Magíster en Demografía y Población, Médico Cirujano.

b. Doctora en Administración, Magíster en Investigación y Docencia Universitaria, Licenciada en Administración.

c. Magister en Ciencias de la Educación Superior, Licenciada en Enfermería. 


\section{INTRODUCCIÓN}

El año 2014 la Organización Mundial de la Salud (OMS) emitió un informe sobre la resistencia antimicrobiana que establece que la resistencia a los antimicrobianos se está convirtiendo en un desafío mundial que amenaza el beneficio clínico de muchos agentes antimicrobianos importantes ${ }^{(1)}$. Una preocupación real en el informe de la OMS fue que la resistencia antimicrobiana es un fenómeno generalizado que puede afectar a cualquier persona sin distinguir edad o país. El año 2015, la OMS publicó un informe acerca de la situación de países a nivel global sobre la respuesta a la resistencia antimicrobiana y destacó que existen brechas importantes en las medidas adoptadas, a pesar de la realización de múltiples actividades y la disposición de los gobiernos para enfrentar este problema ${ }^{(2)}$.

Los profesionales de salud de las instituciones, organizaciones de salud, gobiernos, proveedores de servicios de salud y los investigadores necesitan coordinar esfuerzos y responder a este nuevo desafío global de salud pública mediante la implementación de programas para racionalizar el uso de antibióticos ${ }^{(3,4)}$. Es importante analizar la investigación publicada a nivel mundial sobre la resistencia antimicrobiana, $y$, en específico, la información publicada por autores peruanos. Esos datos de referencia son necesarios para comprender la situación actual de investigación en resistencia antimicrobiana $y$, en consecuencia, nos permitirán en un futuro explorar las redes de colaboración ${ }^{(5)}$ e incluso, plantear nuevas líneas de investigación.

Un método importante y común para determinar la actividad de investigación sobre un determinado tema es el análisis bibliométrico ${ }^{(6,7)}$, que se define como el uso de métodos matemáticos para analizar artículos publicados en términos de cantidad y calidad ${ }^{(8,9)}$. En el análisis bibliométrico se presenta la información con respecto al crecimiento de las publicaciones, la colaboración internacional, los principales países activos, institutos y autores; además, en algunos estudios emplean mapas como método de visualización de indicadores bibliométricos ${ }^{(10)}$.

La recuperación de artículos publicados sobre un tema determinado es el primer paso en la evaluación de la literatura con respecto a un cierto problema o materia y en la construcción de decisiones clínicas basadas en evidencia. Existen algunas bases de datos conocidas que se utilizan para el análisis bibliométrico en el área biomédica, como PubMed, Scopus, Web of Science y
Google Scholar (11). Como base de datos académica, Scopus es superior a PubMed y Web of Science en varios aspectos, particularmente, en el número de cobertura de revistas y análisis de citas ${ }^{(12-15)}$. En la última década, se publicaron una serie de estudios bibliométricos sobre tipos específicos de infecciones y sobre temas médicos específicos de microbiología (16-18). Debido al impacto político, social e incluso económico de la resistencia antimicrobiana a nivel global en la actualidad (19), es fundamental conocer las publicaciones que se han realizado en relación a esta situación en nuestro país. Por lo tanto, este estudio se llevó a cabo para analizar indicadores bibliométricos (20) relacionados con las publicaciones peruanas sobre resistencia antimicrobiana, incluyendo los principales países productivos, instituciones, autores, y revistas.

\section{EL ESTUDIO}

Se realizó un estudio bibliométrico descriptivo que evaluó los datos cuantitativos procedentes de publicaciones científicas ${ }^{(20)}$. La recolección de datos para este estudio se llevó a cabo utilizando la base de datos Scopus ya que es reconocida como la base de datos de citas y resúmenes más grande de la literatura revisada por pares y que cubre una amplia gama de temas (21). Asimismo, ha sido utilizada previamente como única fuente de datos en artículos bibliométricos relacionados a antimicrobianos ${ }^{(22-24)}$.

La estrategia de búsqueda fue similar a la descrita en estudios publicados anteriormente (23-29). Las palabras clave empleadas para la recolección de datos se obtuvieron de artículos publicados sobre resistencia antimicrobiana que aparecen en Scopus (21). Para incluir a un artículo en el estudio se consideró que tuviera, al menos, un autor con filiación peruana y que hubiera sido publicado hasta el 2017, inclusive. La estructura de la búsqueda, adaptada de Sweileh et al. ${ }^{(22)}$, realizada en febrero del 2018 y utilizada para la extracción de datos utilizando la base Scopus, se muestra a continuación: TITLE-ABS-KEY ( "antimicrobial resist*" ) AND PUBYEAR < 2018 AND ( LIMIT-TO ( SRCTYPE , " $\mathrm{"} \mathrm{)} \mathrm{)} \mathrm{AND}$ ( EXCLUDE ( DOCTYPE, "er ") ) AND ( LIMIT-TO ( AFFILCOUNTRY, "Peru" ) ).

El asterisco se utilizó para recuperar todas las posibles palabras correctas, y las comillas para recuperar frases correctas y exactas (22). Se excluyeron las publicaciones tipo fe de erratas ("erratum") y documentos indefinidos según metodología similar al estudio de Swileh et al. ya mencionado ${ }^{(22)}$. La validez de la consulta de búsqueda se confirmó por análisis manual de los artículos citados 
que, en este estudio no mostró ninguna desviación del objetivo requerido para recuperar los documentos sobre resistencia antimicrobiana. Se determinó como lapso de tiempo para la recolección de datos todos los años anteriores al 2018. Los documentos recuperados se limitaron a publicaciones en revistas. Se recuperaron un total de 34 artículos. Los indicadores bibliométricos que se presentan son los principales países productivos, instituciones, autores, y revistas, de acuerdo a los criterios utilizados previamente por Garousi y Mäntylä (30) y por Ding et al. ${ }^{(31)}$.

Para la calidad de las publicaciones, se muestra la clasificación de revistas científicas (SJR) para cada revista, las que se obtuvieron del Scimago Journal Rank (32).

\section{RESULTADOS}

El número promedio de citas por artículo fue de 18 . La mayoría de los documentos recuperados fueron artículos originales (30, que corresponde al $88,2 \%$ ), y de estos, 29 (85,2 \%) estaban escritos en inglés. El idioma español ocupó el segundo lugar con 5 artículos (20,8\%). Las publicaciones peruanas sobre resistencia antimicrobiana en Scopus aumentaron considerablemente a partir del año 2015, llegando a un récord de 11 en el año 2017 (Figura 1). El artículo más antiguo acerca de resistencia antimicrobiana que contó con participación peruana se publicó en Scopus en 1992. Este reporte discutió las prácticas de lavado de manos y evaluó la resistencia y densidad de flora bacteriana en dos unidades pediátricas en Lima ${ }^{(33)}$.

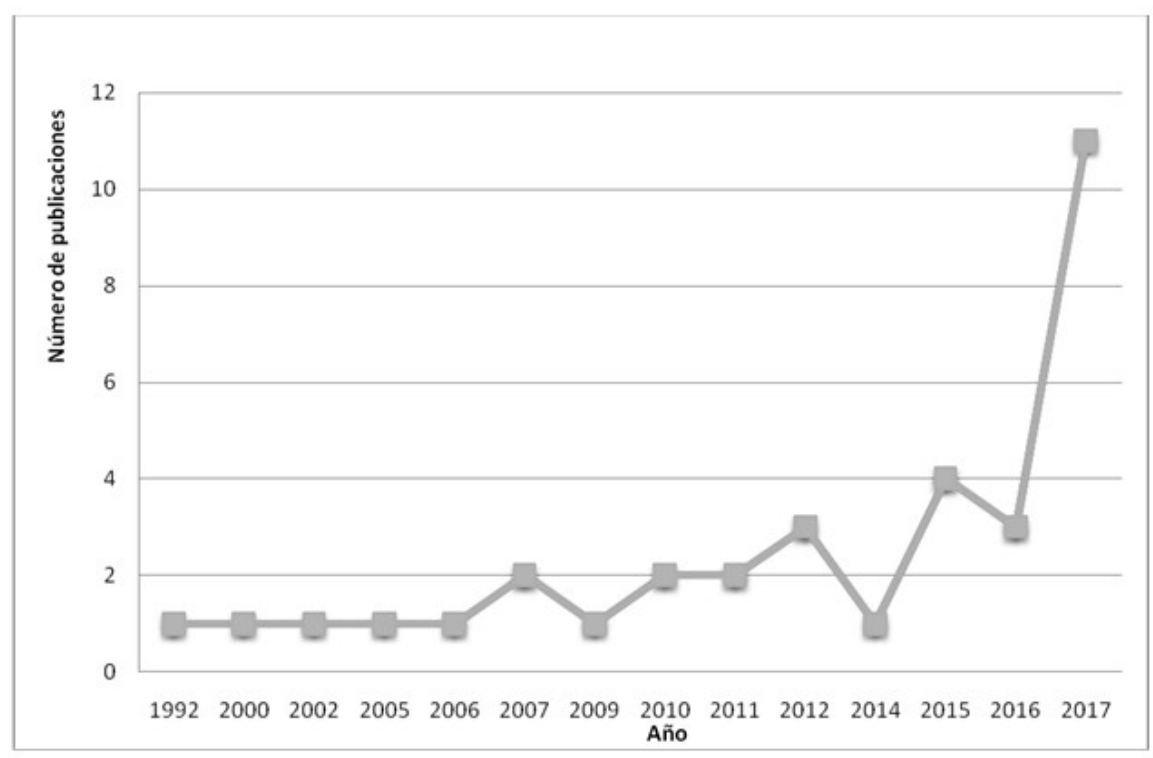

Figura 1. Distribución temporal de publicaciones peruanas sobre resistencia antimicrobiana en Scopus (1992-2017)

Al analizar las citaciones de las publicaciones, se encontró que el número de citas por artículo por año mostró una relación inversa con el tiempo a partir del año 2007, lo que indica que los artículos más antiguos se continúan citando a través del tiempo.

El análisis de los términos relevantes en los títulos de los artículos sobre resistencia antimicrobiana mostró que "E. coli" fue el término más frecuente, seguido de "Bartonella", "Vibrio cholerae", "Salmonella", "Staphylococcus aureus" y neumococo.
Las revistas con mayor número de artículos publicados sobre la resistencia antimicrobiana se muestran en la tabla 1 . Diecisiete $(50 \%)$, aparecieron en las primeras ocho revistas listadas en la dicha tabla. De estas, la Revista Peruana de Biología, editada por la Facultad de Ciencias Biológicas de la Universidad Nacional Mayor de San Marcos, es la que consigna el mayor número de artículos publicados sobre el tema. 
Tabla 1. Revistas que han publicado sobre resistencia antimicrobiana en SCOPUS (1992-2017)

\begin{tabular}{|c|c|c|c|}
\hline Revista & $\begin{array}{l}\text { Número de } \\
\text { artículos } \\
\text { publicados }\end{array}$ & $\begin{array}{c}\text { Número } \\
\text { total de } \\
\text { citaciones }\end{array}$ & $\begin{array}{l}\text { SJR } \\
2016\end{array}$ \\
\hline Revista Peruana de Biología & 3 & 3 & 0,1 \\
\hline Transactions of the Royal Society of Tropical Medicine and Hygiene & 2 & 28 & 1,0 \\
\hline International Journal of Antimicrobial Agents & 2 & 31 & 1,5 \\
\hline Journal of Global Antimicrobial Resistance & 2 & 0 & 0,5 \\
\hline World Journal of Emergency Surgery & 2 & 20 & 0,8 \\
\hline Revista Peruana de Medicina Experimental y Salud Pública & 2 & 4 & 0,2 \\
\hline International Journal of Medical Microbiology & 2 & 12 & 1,6 \\
\hline Brazilian Journal of Infectious Diseases & 2 & 0 & 0,6 \\
\hline Microbiome & 1 & 7 & 6,2 \\
\hline Journal of Tropical Pediatrics & 1 & 15 & 0,7 \\
\hline Journal of Infection & 1 & 5 & 2,0 \\
\hline Sexually Transmitted Diseases & 1 & 21 & 1,7 \\
\hline BMC Infectious Diseases & 1 & 22 & 1,5 \\
\hline BMC Clinical Pharmacology & 1 & 28 & 0 \\
\hline BMC Public Health & 1 & 8 & 1,3 \\
\hline Revista Panamericana de Salud Pública & 1 & 17 & 0,4 \\
\hline Scandinavian Journal of Infectious Diseases & 1 & 6 & 0 \\
\hline Annals of Surgery & 1 & 124 & 4,2 \\
\hline AJIC: American Journal of Infection Control & 1 & 42 & 1,0 \\
\hline PLoS ONE & 1 & 0 & 1,2 \\
\hline Clinical Microbiology and Infection & 1 & 1 & 2,0 \\
\hline Surgical Infections & 1 & 0 & 0,5 \\
\hline BMC Medicine & 1 & 0 & 3,8 \\
\hline Military Medicine & 1 & 0 & 0,3 \\
\hline The Lancet Respiratory Medicine & 1 & 17 & 6,3 \\
\hline
\end{tabular}

Fuente: SCOPUS

Los artículos recuperados fueron publicados por 104 países diferentes distribuidos en todas las regiones del mundo. La lista de los diez principales países productores incluye Estados Unidos (17), España (9), Bélgica (8), Italia (7), Suiza (7), Reino Unido (7), Colombia (6), Egipto (6), México (6) y Sudáfrica (6). Las cinco instituciones más productivas fueron Universidad Peruana Cayetano Heredia (13), Universidad Nacional Mayor de San Marcos (6), Universitat de Barcelona (5), University of Witwastersrand (5), y el Hospital Clinic Barcelona (5). Los autores más activos fueron Ochoa TJ (6), García C (4), Jacobs J (4), Ruiz J (4) y Abu-Zidan FM (3).

\section{DISCUSIÓN}

El estudio tuvo como objetivo realizar una descripción bibliométrica de las publicaciones peruanas sobre resistencia antimicrobiana. Luego de una revisión de la literatura se encontró que se han publicado pocos estudios sobre este punto (22), y los que se han realizado están redactados en inglés y, además, aparecen en publicaciones editadas en este idioma. En nuestro conocimiento, este es el primer estudio bibliométrico sobre resistencia antimicrobiana publicado en español.

El gran aumento en el número de publicaciones sobre resistencia antimicrobiana en los últimos años refleja la preocupación real de la comunidad científica y profesionales de la salud sobre este tema y el hecho de que el problema se está extendiendo por todo el mundo ${ }^{(1)}$. 
Las principales revistas en las que aparecen artículos peruanos sobre resistencia antimicrobiana son publicaciones reconocidas y prestigiosas en el campo de la microbiología y las enfermedades infecciosas. En este ámbito, la lista está encabezada por una revista peruana que cuenta con tres artículos publicados sobre resistencia antimicrobiana realizados en nuestro país. Es frecuente encontrar en estudios de productividad científica de un país, que la revista de mayor publicación es una revista del propio país. A este comportamiento se le conoce como endogamia de la producción científica ${ }^{(34)}$.

Se observa que ninguna de las revistas principales pertenece al área de enfermería. Este hallazgo llama la atención y se relaciona con un artículo donde encontraron que solo alrededor del $1 \%$ de los muchos artículos sobre resistencia a los antimicrobianos estaban dentro de la literatura de enfermería, lo que es sorprendente y alarmante, ya que las enfermeras son el mayor grupo de profesionales de la salud que interactúan con el público y pacientes ${ }^{(35)}$.

Las enfermeras tienen el conocimiento de los estándares de seguridad en la atención de los pacientes y la responsabilidad de cumplirlos, asimismo de una función única e integral, desde la preparación, administración y prescripción de antibióticos hasta el control de los efectos de los antimicrobianos. Además, cumplen un papel clave en la prevención y el control. Este rol también abarca la práctica asistencial y la interacción con pacientes y familias, así como su participación en los procesos de promoción, prevención, recuperación y rehabilitación de la salud. Por ello, es necesario revisar y discutir más a fondo cómo la enfermería puede ser sinérgica en la lucha contra la resistencia a los antimicrobianos.

Las dos instituciones peruanas con mayor productividad en este estudio coinciden con las que cuentan con el mayor número de publicaciones a nivel nacional indexadas en Scopus.

Nuestro estudio tiene la ventaja de ser el primero en ofrecerunadescripciónbibliométrica delaspublicaciones peruanas sobre la resistencia antimicrobiana. Una de nuestras fortalezas para el estudio fue utilizar una metodología previamente utilizada en varios artículos bibliométricos ${ }^{(22)}$. Sin embargo, es posible que algunos artículos sobre resistencia antimicrobiana realizada por o en colaboración por autores peruanos puedan no haber sido incluidos. Además, también puede ocurrir que algunas revistas biomédicas que publicaron artículos de autores peruanos sobre resistencia antimicrobiana no se encuentran indexadas en Scopus, sobre todo, revistas en español. Sin embargo, Scopus es considerada una fuente completa, precisa y superior para estudios bibliométricos ${ }^{(13-15)}$. Artículos adicionales pueden haber sido recuperados al incluir otras bases de datos y repositorios como Web of Science, SciELO, LILACS, e incluso el Repositorio Nacional Digital ALICIA.

En conclusión, se realizó un estudio bibliométrico descriptivo que evaluó las publicaciones peruanas sobre resistencia microbiana. Se encontró que, si bien se han publicado pocos estudios relacionados a resistencia antimicrobiana, hubo un aumento dramático en el número de publicaciones peruanas sobre resistencia antimicrobiana en los últimos tres años, lo que concuerda con un reporte reciente de la Organización Mundial de la Salud en relación a la creciente amenaza para la salud pública de la resistencia antimicrobiana y que genera amplia preocupación para los países y múltiples sectores en todo el mundo ${ }^{(1)}$.

Las publicaciones peruanas sobre resistencia antimicrobiana en Scopus se originaron en diferentes regiones del mundo, incluidas América del Norte, Europa y América Latina. La mayor parte de los artículos recuperados se publicaron en revistas prestigiosas de biología, medicina, microbiología y en revistas relacionadas con el control de infecciones.

Contribuciones de autoría: EEP, FLJ y GJVC participaron en la concepción, diseño y redacción del manuscrito de manera conjunta. Todos los autores aprobaron la versión final a publicar, y asumen la responsabilidad frente a todos los aspectos del manuscrito.

\section{REFERENCIAS BIBLIOGRÁFICAS}

1. World Health Organization. Antimicrobial resistance: global report on surveillance [internet].Geneva: WHO; 2014. Disponible en: http://apps.who.int/iris/ bitstream/10665/112642/1/9789241564748_eng.pdf.

2. World Health Organization. Worldwide country situation analysis: Response to antimicrobial resistance [internet]. Geneva: WHO; 2015. Disponible en: http://apps.who.int/ iris/bitstream/10665/163468/1/9789241564946_eng.pdf

3. Laxminarayan R, Duse A, Wattal C, Zaidi AK, Wertheim HF, Sumpradit N, et al. Antibiotic resistance-the need for global solutions. Lancet Infect Dis. 2013;13(12):1057-98.

4. Ganguly NK, Arora NK, Chandy SJ, Fairoze MN, Gill JP, Gupta $\mathrm{U}$, et al. Rationalizing antibiotic use to limit antibiotic resistance in India. Indian J Med Res. 2011;134: 281-94.

5. Huamaní C, González AG, Curioso WH, Pacheco-Romero J. Redes de colaboración y producción científica sudamericana en medicina clínica, ISI Current Contents 2000-2009. Rev Med Chil. 2012;140(4):466-75.

6. Bornmann L, Mutz R. Growth rates of modern science: a bibliometric analysis based on the number of publications and cited references. J Assoc Inf Sci Technol. 2015;66: 2215-22.

7. Thompson DF, Walker CK. A descriptive and historical review 
of bibliometrics with applications to medical sciences. Pharmacotherapy. 2015;35(6):551-9.

8. Broadus R. Toward a definition of "bibliometrics". Scientometrics. 1987;12(5-6):373-9.

9. Okubo Y. Bibliometric indicators and analysis of research systems: methods and examples. Paris: OCDE; 1997.

10. Cobo MJ, López-Herrera AG, Herrera-Viedma E, Herrera F. Science mapping software tools: Review, analysis and cooperative study among tools. J Am Soc Inf Sci. 2011;62(7):1382-1402.

11. Halevi G, Moed H, Bar-Ilan J. Suitability of Google Scholar as a source of scientific information and as a source of data for scientific evaluation-Review of the literature. J Informetr. 2017, 11(3):823-834.

12. Tadmouri GO, Bissar-Tadmouri N. A major pitfall in the search strategy on PubMed. Saudi Med J 2004; 25 (1): 7-10.

13. Falagas ME, Pitsouni EI, Malietzis GA, Pappas G. Comparison of PubMed, Scopus, Web of Science, and Google Scholar: strengths and weaknesses. FASEB J. 2008;22(2):338-42.

14. Kulkarni AV, Aziz B, Shams I, Busse JW. Comparisons of citations in Web of Science, Scopus, and Google Scholar for articles published in general medical journals. JAMA. 2009;302(10):1092-6.

15. de Granda-Orive JI, Alonso-Arroyo A and Roig-Vazquez F. Which data base should we use for our literature analysis? Web of Science versus SCOPUS. Arch Bronconeumol 2011; 47(4): 213.

16. Macías-Chapula CA, Mijangos-Nolasco A. Bibliometric analysis of AIDS literature in Central Africa. Scientometrics. 2002;54(2):309-17.

17. Sweileh WM, Al-Jabi SW, Abuzanat A, Sawalha AF, AbuTaha AS, Ghanim MA, Zyoud SH. Assessment of research productivity of Arab countries in the field of infectious diseases using Web of Science database. Infect Dis Poverty. 2015;4(1):2.

18. Ramos JM, Padilla S, Masia M, Gutierrez F. A bibliometric analysis of tuberculosis research indexed in PubMed, 19972006. Int J Tuberc Lung Dis. 2008;12(12):1461-8.

19. Ramón-Pardo P, Sati H, Galas M. Enfoque de Una Salud en las acciones para enfrentar la resistencia a los antimicrobianos desde una óptica latinoamericana. Rev Peru Med Exp Salud Publica. 2018;35(1):103-9.

20. Bordons M, Zulueta MA. (Evaluation of the scientific activity through bibliometric indices). Rev Esp Cardiol. 1999;52(10):790-800.

21. Md Khudzari J, Kurian J, Tartakovsky B, Vijaya Raghavan GS. Bibliometric analysis of global research trends on microbial fuel cells using Scopus database. Biochem Eng J. 2018; 136: 51-60.

22. Sweileh WM, Shraim NY, Al-Jabi SW, Sawalha AF, AbuTaha AS, Zyoud SH. Bibliometric analysis of global scientific research on carbapenem resistance (1986-2015). Ann Clin Microbiol Antimicrob. 2016;15(1):56.

23. Sweileh WM, Al-Jabi SW, Zyoud SH, Sawalha AF. Bronchial asthma and chronic obstructive pulmonary disease: research activity in Arab countries. Multidiscip Respir Med. 2014;9(1):38.

24. Bielsa S, Porcel JM. Trends in pleural effusion research. Pleura. 2016;3:237399751664655.

25. Zyoud SH, Al-Jabi SW, Sweileh WM, Awang R. Contribution of Arab countries to pharmaceutical wastewater literature: a bibliometric and comparative analysis of research output.

Ann Occup Environ Med. 2016;28:28

26. Zyoud SH. Global research trends of Middle East respiratory syndrome coronavirus: a bibliometric analysis. BMC Infect Dis. 2016;16(1):255.

27. Zyoud SH. Dengue research: a bibliometric analysis of worldwide and Arab publications during 1872-2015. Virol J. 2016;13(1):78.

28. Zyoud SH, Al-Jabi SW, Sweileh WM, Al-Khalil S, Alqub M, Awang R. Global methaemoglobinaemia research output (1940-2013): a bibliometric analysis. Springerplus. 2015; 4: 626.

29. Zyoud SH, Al-Jabi SW, Sweileh WM, Awang R, Waring WS. Bibliometric profile of the global scientific research on methanol poisoning (1902-2012). J Occup Med Toxicol. 2015;10:17.

30. Garousi V, Mäntylä MV. Citations, research topics and active countries in software engineering: A bibliometrics study. Computer Science Review 2016; 19:56-77.

31. Ding ZQ, Ge JP, Wu XM, Zheng XN. Bibliometrics evaluation of research performance in pharmacology/pharmacy: China relative to ten representative countries. Scientometrics. 2013; 96 (3): 829-844.

32. SCImago. Scimago Journal and country rank [internet]. 2016. Disponible en: http://www.scimagojr.com/journalrank.php.

33. Larson EL, McGinley KJ, Foglia A, Leyden JJ, Boland N, Larson $J$, et al. Handwashing practices and resistance and density of bacterial hand flora on two pediatric units in Lima, Peru. Am J Infect Control. 1992;20(2):65-72.

34. Fernández Cano A. Producción educativa española en el Social Sciences Citation Index (1998-2009) II. Rev Esp Pedadog. 2011;69(250):427-444.

35. Ellen ME, Hughes $F$, Shach $R$, Shamian J. How nurses can contribute to combating antimicrobial resistance in practice, research and global policy. Int J Nurs Stud. 2017;71: A1-A3.

Fuentes de financiamiento:

Este artículo ha sido financiado por los autores.

Conflictos de interés:

Los autores declaran no tener ningún conflicto de interés.

\section{Correspondencia:}

Elizabeth Espinoza-Portilla

Dirección: Calle Martín Dulanto 101, Urb. San Antonio, Miraflores. Lima, Perú.

Teléfono: 998796750.

Correo electrónico: espinoza.portilla@gmail.com

Recibido: 22 de mayo de 2018

Evaluado: 26 de junio de 2018 Aprobado: 15 de octubre de 2018

(c) La revista. Publicado por Universidad de San Martín de Porres, Perú. (c) Br Licencia de Creative Commons Artículo en acceso abierto bajo términos de Licencia Creative Commons Atribución 4.0 Internacional. (http://creativecommons.org/licenses/by/4.0/)

\section{ORCID iDs}

Elizabeth Espinoza-Portilla

Flor Lioo-Jordán

Gladis J. Villanueva-Cadenas https://orcid.org/0000-0002-5068-5357 https://orcid.org/0000-0002-8448-2718 https://orcid.org/0000-0002-5618-0114 